\title{
OPEN DNA metabarcoding uncovers fungal diversity in soils of protected and non-protected areas on Deception Island, Antarctica
}

\author{
Luiz Henrique Rosa ${ }^{1 凶}$, Thamar Holanda da Silva ${ }^{1}$, Mayara Baptistucci Ogaki ${ }^{1}$, \\ Otávio Henrique Bezerra Pinto ${ }^{2}$, Michael Stech ${ }^{3}$, Peter Convey ${ }^{4}$, Micheline Carvalho-Silva ${ }^{5}$, \\ Carlos Augusto Rosa ${ }^{1} \&$ Paulo E. A. S. Câmara ${ }^{5}$
}

We assessed soil fungal diversity at two sites on Deception Island, South Shetland Islands, Antarctica using DNA metabarcoding analysis. The first site was a relatively undisturbed area, and the second was much more heavily impacted by research and tourism. We detected 346 fungal amplicon sequence variants dominated by the phyla Ascomycota, Basidiomycota, Mortierellomycota and Chytridiomycota. We also detected taxa belonging to the rare phyla Mucoromycota and Rozellomycota, which have been difficult to detect in Antarctica by traditional isolation methods. Cladosporium sp., Pseudogymnoascus roseus, Leotiomycetes sp. 2, Penicillium sp., Mortierella sp. 1, Mortierella sp. 2, Pseudogymnoascus appendiculatus and Pseudogymnoascus sp. were the most dominant fungi. In addition, 440,153 of the total of 1,214, 875 reads detected could be classified only at the level of Fungi. In both sampling areas the DNA of opportunistic, phytopathogenic and symbiotic fungi were detected, which might have been introduced by human activities, transported by birds or wind, and/or represent resident fungi not previously reported from Antarctica. Further long-term studies are required to elucidate how biological colonization in the island may be affected by climatic changes and/or other anthropogenic influences.

The pristine environments of Antarctica are used as field laboratories to support taxonomic, ecological, evolutionary and biotechnological studies. Antarctic environments experience multiple extreme conditions including low temperatures, acidic and alkaline $\mathrm{pH}$, ultra-oligotrophic conditions, freeze-thaw cycles, salinity stress, desiccation, wind abrasion and high radiation levels ${ }^{1}$ and, for these reasons, offer unique opportunities to study the diversity of fungi ${ }^{2}$.

In the latter part of the Twentieth Century, the Antarctic Peninsula region was one of the regions of the planet most affected by climatic changes. Deception Island, located in the South Shetland Islands is one of very few active volcanoes in the Antarctic Treaty area. Two summer-only research stations are presently active on the island (the Argentinian Decepción and Spanish Gabriel de Castilla). In addition, a shore-based whaling station operated in Whalers Bay in the early Twentieth Century ${ }^{3}$. The combination of unique geology, history, biota and aesthetic values, as well as the active presence of multiple national operators, underlie the designation of the entire island as an Antarctic Specially Managed Area (ASMA 4). In addition, Deception Island includes two Antarctic Specially Protected Areas (ASPAs), designated as ASPAs 140 (terrestrial, formed of multiple sub-sites) and 145 (marine). Deception Island is one of the best-known locations in Antarctica, visited by both researchers and tourists ${ }^{4}$, with more than 55,489 tourists visiting the island in the summer of 2018-2019 (https://iaato.org/ tourism-statistics-327mnsyd), which generates pressure on its ecosystems. The island is an exceptional location even within Antarctica, as it is a young volcanic island formed less than $100 \mathrm{kya}^{5}$ and still in the process of biological colonization.

\footnotetext{
${ }^{1}$ Laboratório de Microbiologia Polar \& Conexões Tropicais, Departamento de Microbiologia, Instituto de Ciências Biológicas, Universidade Federal de Minas Gerais, P. O. Box 486, Belo Horizonte, MG CEP 31270-901, Brazil. ${ }^{2}$ Departamento de Biologia Celular, Universidade de Brasília, Brasília, Brazil. ${ }^{3}$ Naturalis Biodiversity Center, Leiden, The Netherlands. "British Antarctic Survey, NERC, High Cross, Madingley Road, Cambridge CB3 OET, UK. ${ }^{5}$ Departamento de Botânica, Universidade de Brasília, Brasília, Brazil. ${ }^{\circledR}$ email: Ihrosa@icb.ufmg.br
} 


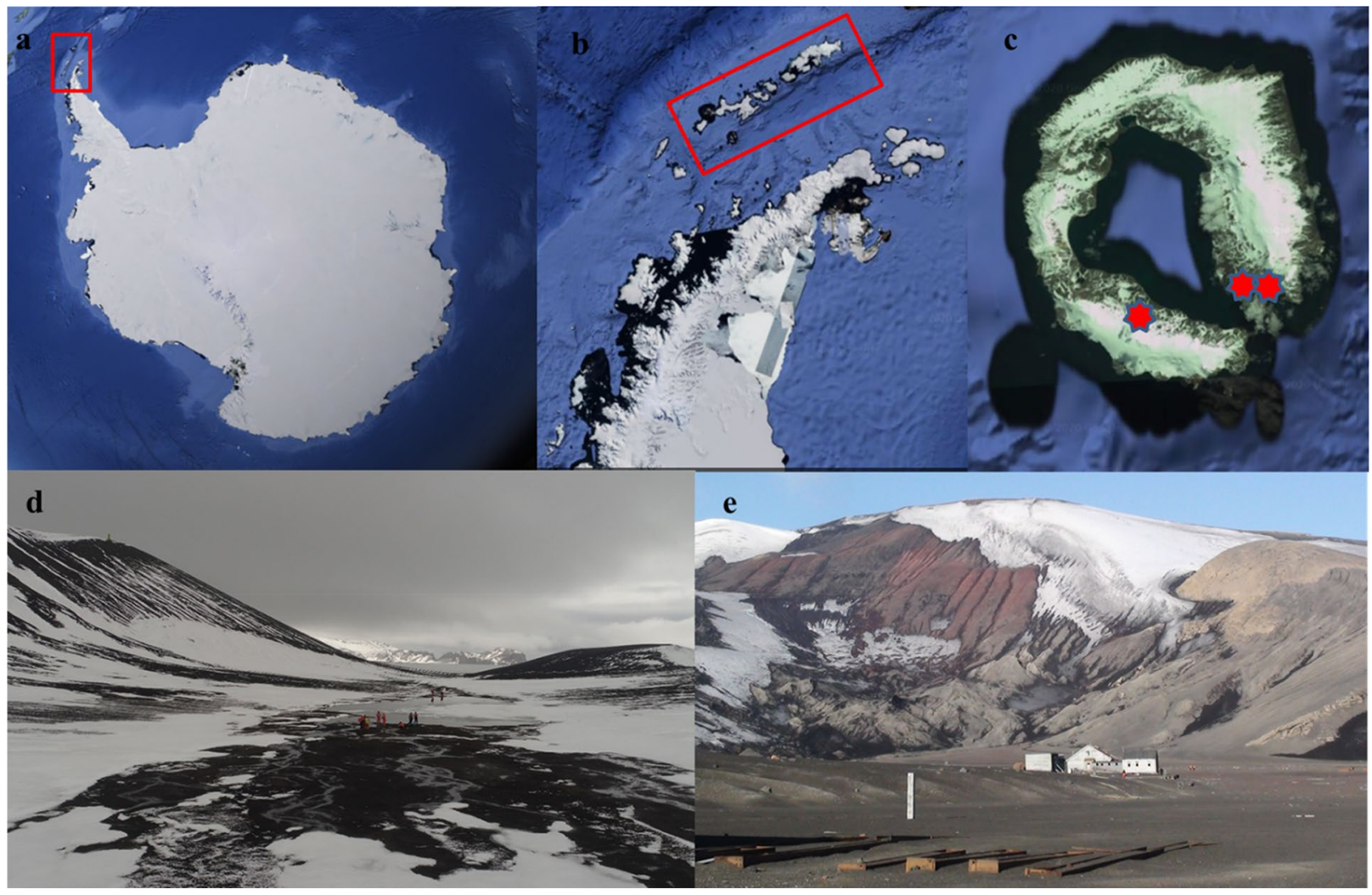

Figure 1. Satellite images (a-c) (obtained in Google Earth Pro, 2019) and sites were the soil where sampled. (a) Antarctic Continent with the northern Antarctic Peninsula inside the red rectangle, (b) Antarctic Peninsula with the South Shetland Islands archipelago inside the red rectangle, (c) Deception Island with the sites *ASPA 140 and ${ }^{* *}$ Whalers Bay, (d) Antarctic Specially Protected Area 140 subsite B (protected area close to Crater Lake-62 $06^{\prime} 08.6^{\prime \prime} \mathrm{S} ; 57^{\circ} 55^{\prime} 10.4^{\prime \prime} \mathrm{W}$ ), and (e) Whalers Bay (non-protected area, WB-62 $58^{\prime} 52.0^{\prime \prime} \mathrm{S} ; 60^{\circ} 39^{\prime}$ 52.9" W). Photos (d,e) by L.H. Rosa.

The majority of mycological studies in Antarctica to date have focused on cultivable species, mainly represented by taxa of the phylum Ascomycota and its anamorphs, followed by Basidiomycota, Mortierellomycota, Mucoromycota, Chytridiomycota and Glomeromycota ${ }^{2}$. In Antarctica, different fungal assemblages contribute to complex ecological networks, including saprophytic, mutualistic and parasitic taxa, all of which are able to survive under various extreme environmental conditions ${ }^{6,7}$. However, despite the recognized importance of fungal diversity in Antarctica, few studies have applied metabarcoding approaches using high throughput sequencing (HTS). The present study aimed to characterize and compare fungal diversity assessed using metabarcoding in soil at two sites on Deception Island, (1) a relatively undisturbed site within the terrestrial Antarctic Specially Protected Area (ASPA) 140 and (2) a disturbed site in Whalers Bay subject to considerable visitor pressure and hence greater human impact.

\section{Methods}

Soil sampling. Soil samples were collected from two sites on Deception Island, South Shetland Islands (Fig. 1). The first was within an Antarctic Specially Protected Area (ASPA) close to Crater Lake [ASPA 140, subsite B], which has relatively low impact from researchers and is not accessible for tourism. The second site was in Whalers Bay, which includes the area of the historical whaling station and former UK research station on the island, and is formally declared a Historic Monument. It is one of the most popular visitor sites in Antarctica for both tourists and national operator personnel. The distance between the two sites is approximately $5 \mathrm{~km}$. Superficial soil samples (approximately $5 \mathrm{~cm}$ depth and ca $250 \mathrm{~g}$ each) were collected using sterile spatulas and immediately placed in sterilized WhirlPak bags (Sigma-Aldrich, USA) kept at $-20{ }^{\circ} \mathrm{C}$ until processing. Seven (non-composite) samples from each site (obtained a minimum of $10 \mathrm{~m}$ from each other) were collected for use in DNA studies, totaling 14 samples in total.

DNA extraction and analysis, and fungal identification. Total DNA was extracted from environmental samples using the QIAGEN Power Soil Kit, following the manufacturer's instructions. Extracted DNA was used as template for generating PCR-amplicons. The internal transcribed spacer 2 (ITS2) of the nuclear ribosomal DNA was used as a DNA barcode for molecular species identification ${ }^{8,9}$. PCR-amplicons were generated using the universal primers ITS3 and ITS $4^{10}$ and were sequenced by high-throughput sequencing at Macrogen 

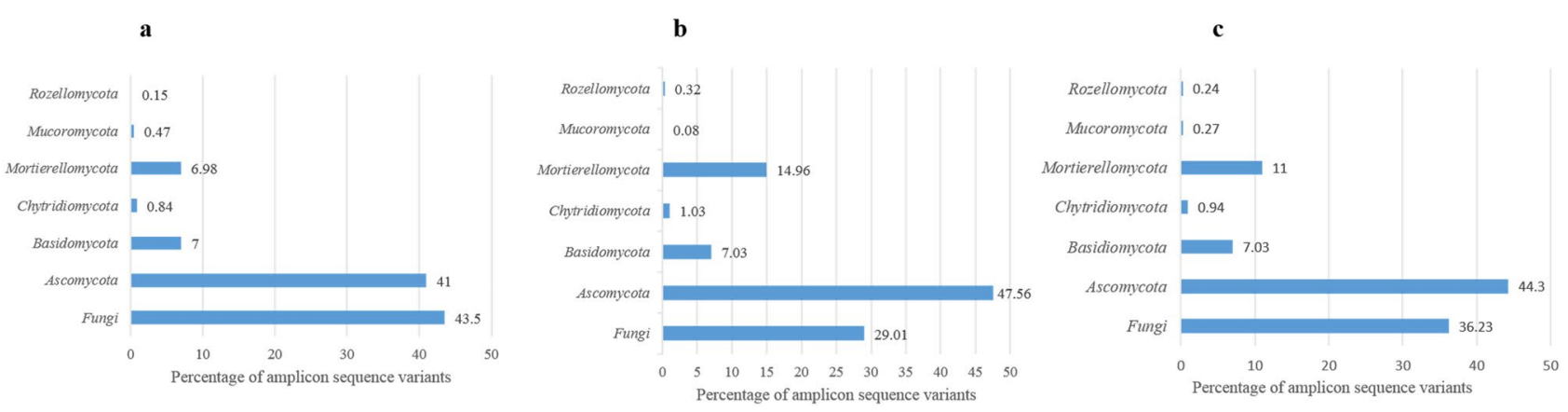

Figure 2. Percentage of fungal amplicon sequence variants (ASVs) at phylum level identified from soil of Deception Island, South Shetland Islands. (a) Fungal assemblage of soil in ASPA 140 (non-impacted site), (b) fungal assemblage of soil in Whalers Bay (impacted site), and (c) total soil fungal community of both sites.

Inc. (South Korea) on an Illumina MiSeq sequencer, using the MiSeq Reagent Kit v3 (600-cycle) following the manufacturer's protocol.

Raw fastq files were filtered using BBDuk version 38.34 (BBMap-Bushnell B.—sourceforge.net/projects/ bbmap/) to remove Illumina adapters, known Illumina artifacts, and PhiX Control v3 Library. Quality read filtering was carried out using Sickle version 1.33 -q 30-1 50 ${ }^{11}$, to trim $3^{\prime}$ or $5^{\prime}$ ends with low Phred quality score, and sequences smaller than $50 \mathrm{bp}$ were also discarded. Remaining sequences were imported to QIIME2 version 2019.10 (https://qiime2.org/) for bioinformatics analyses ${ }^{12}$. The qiime2-dada2 plugin is a complete pipeline that was used to filter, dereplicate, turn paired-end fastq files into merged, and remove chimeras ${ }^{13}$. Taxonomic assignments were determined for amplicon sequence variants (ASVs) using qiime2-feature-classifier ${ }^{14}$ classify-sklearn against the UNITE fungal ITS database version $7.2^{15}$ and trained with Naive Bayes classifier and a confidence threshold of $98.5 \%$.

Many factors, including extraction, PCR and primer bias, can affect the number of reads ${ }^{16}$, and thus lead to misinterpretation of abundance ${ }^{17}$. However, Giner et al. ${ }^{18}$ concluded that such biases did not affect the proportionality between reads and cell abundance, implying that more reads are linked with higher abundance ${ }^{19,20}$. Therefore, for comparative purposes we used the number of reads as a proxy for relative abundance.

Fungal diversity and distribution. To quantify species diversity, richness and dominance, we used the following indices: (1) Fisher's $\alpha$, (2) Margalef's and (3) Simpson's, respectively. The numbers of reads of each amplicon sequence variant (ASV) were used to quantify the fungal taxa present in the soils sampled, where fungal ASVs $>6000$ were considered dominant and $\leq 1000$ minor components (rare) within the fungal community. Species accumulation curves were assessed using the Mao Tao index. All diversity index calculations were performed using PAST, version $1.90^{21}$. Results were obtained with $95 \%$ confidence, and bootstrap values were calculated from 1000 iterations. Venn diagrams were prepared according to Bardou et al. ${ }^{22}$ to illustrate the comparison of fungal assemblages present in the two sampling areas.

\section{Results}

Fungal taxonomy. We detected 346 soil fungal amplicon sequence variants (ASVs) in the samples from the two sites on Deception Island (Suppl. Table 1). Ascomycota, Basidiomycota, Mortierellomycota and Chytridiomycota dominated the fungal assemblages of both sites at phylum level (Fig. 2). We also detected representatives of the generally rare phyla Mucoromycota and Rozellomycota, which occurred at moderate dominance in both sites. The ASVs identified as Cladosporium sp., Pseudogymnoascus roseus, Leotiomycetes sp. 2, Penicillium sp., Mortierella sp. 1, Mortierella sp. 2, Pseudogymnoascus appendiculatus and Pseudogymnoascus sp. were most dominant at genus/species level (with $>30,000$ reads). A further 65 ASVs were moderately dominant ( $>1000$ reads). Twenty-three fungal ASVs could be assigned to only higher hierarchical levels (phylum, class, order or family) when compared with known DNA sequences deposited in the UNITE DNA database ${ }^{15}$ and might represent taxa above the species level new to science and new records for Antarctica. In addition, 440,153 of the total of $1,214,875$ reads detected $(262,844$ in the ASPA and 177,309 in Whalers Bay) could only be classified at the level of Fungi.

Fungal diversity. The Mao Tao rarefaction curves reached asymptote for both fungal assemblages (Fig. 3), indicating that the data provided a good description of the diversity present. The fungal assemblages of both sites displayed high diversity, richness, and dominance indices (Table 1) when compared with studies of cultivable fungi present in Antarctic soils ${ }^{23,24}$. That of Whalers Bay had the highest values of each index.

Of the fungal ASVs characterized, 103 were present only in ASPA 140, 117 in Whalers Bay, with 138 common to both (Fig. 4a), indicating that a small majority of the diversity at both sites was shared between them. The ecological assemblage profiles of exclusive or shared fungi between the two sites did not display significant differences. In both sites the DNA of both cosmopolitan and Antarctic endemic fungi was detected (Suppl. Table 1).

When the dominant fungi (> 6000 reads) were compared between the two sites (Fig. 4b), Malassezia restricta, Mortierella fimbricystis and M. antarctica occurred only in the ASPA samples, and Leucosporidiella creatinivora, 
a

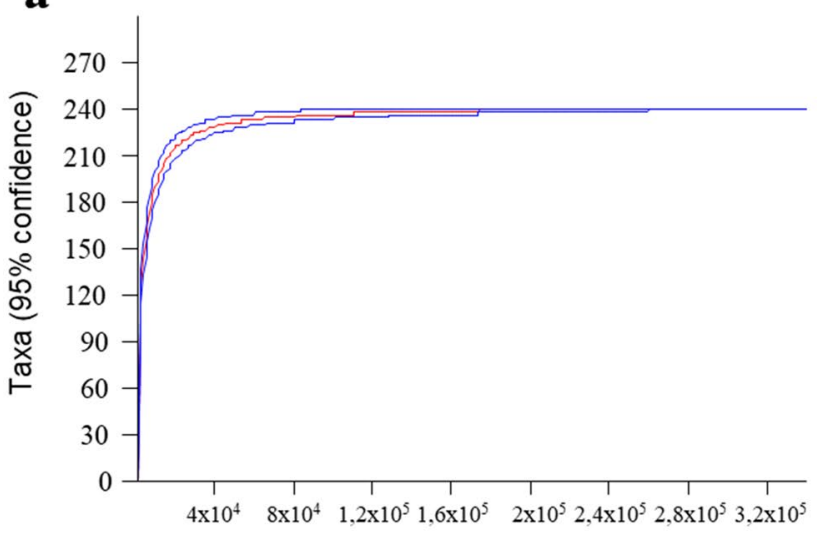

Specimens

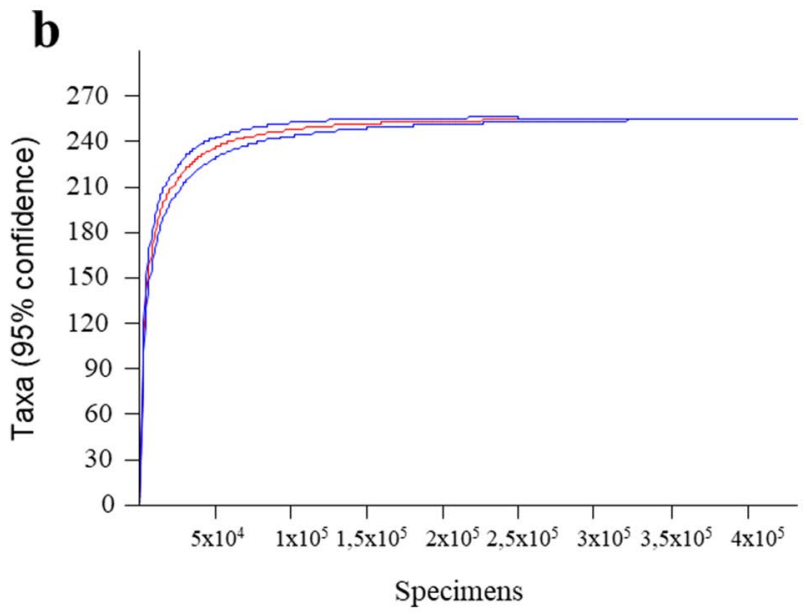

Figure 3. Rarefaction curves for samples from (a) ASPA 140 sampling site and (b) Whalers Bay site (impacted area) on Deception Island, South Shetland Islands. Blue lines represent confidence limits.

\begin{tabular}{|l|l|l|l|l|}
\hline Indices & ASPA $\mathbf{1 4 0}^{\mathbf{b}}$ & Whalers Bay & Gonçalves et al. ${ }^{23 c}$ & Gomes et al. ${ }^{24 c}$ \\
\hline Numbers of ASVs ${ }^{\mathrm{a}}$ & 240 & 255 & $15^{\text {d }}$ & $34^{\text {d }}$ \\
\hline Fisher $\alpha$ & 25.23 & 26.25 & 10.26 & 4.45 \\
\hline Margalef & 18.76 & 19.57 & 3.97 & 3.61 \\
\hline Simpson & 0.91 & 0.93 & 0.85 & 0.95 \\
\hline
\end{tabular}

Table 1. Diversity indices of fungal assemblages present in soils of the Antarctic Specially Protected Area (ASPA) 140 and Whalers Bay sampling sites on Deception Island, as indicated by numbers of amplicon sequence variants (ASVs) and compared with diversity results of cultivable fungi present in soils of Antarctica. ${ }^{\mathrm{a}} \mathrm{ASV}$ = amplicon sequence variants. ${ }^{\mathrm{b}} \mathrm{ASPA}=$ Antarctic Specially Protected Area. ${ }^{\mathrm{c}}$ Gonçalves et al. ${ }^{23}$ and Gomes et al. ${ }^{24}$ represent diversity results of cultivable fungi. ${ }^{\mathrm{d} N u m b e r}$ of fungal taxa detected.

Cleistothelebolus nipigonensis, Thelebolus globosus, Colletotrichum sp. 1 and Leotiomycetes sp. 2 only in the Whalers Bay samples. Pseudogymnoascus roseus, P. appendiculatus, Pseudogymnoascus sp., Cladosporium sp., Mortierella sp. 2 and Penicillium sp. were present in both areas.

The patterns of occurrence of rare taxa (those detected with reads $\leq 1000$ ) in both sites indicated similarities in their ecological status between the assemblages, with the presence of human and animal opportunistic and plant pathogenic taxa (Table 2). In the heavily human impacted Whalers Bay a proportion of the identifiable fungi detected have previously been reported as being opportunistically associated with humans and animals (16 taxa) or phytopathogenic (16). In the soil of ASPA 140, 13 human and animal opportunistic and 12 phytopathogenic taxa were detected. Aspergillus sydowii, Curvularia lunata, Malassezia dermatis, M. globosa, M. restricta, $M$. sympodialis, Rhodotorula mucilaginosa and Trichosporon asahii (human and animal associated), and Aspergillus niger, Colletotrichum annellatum, Curvularia lunata, Gibberella tricincta, G. zeae, Herpotrichia juniper, Nigrospora oryzae, Thanatephorus cucumeris and Cleistothelebolus nipigonensis (phytopathogenic) were detected in both sites. We also detected the presence of DNA of 11 lichenized fungi, of which five (Lecidea cancriformis, Psoroma tenue, Trimmatothelopsis smaragdula, Verrucaria alpicola and V. margacea) occurred in both sites.

\section{Discussion}

Fungal taxonomy and diversity. In Antarctica, around 1000 fungal species have been described through studies of the macro- and/or micromorphology of colonies and fruiting bodies, and DNA sequencing of mycelia of cultivable fungi ${ }^{25}$. However, according to Amann et al. ${ }^{26}$ and Rappe and Giovannoni ${ }^{27}$, just $0.01-1 \%$ of the microbial life present in a given habitat can be characterized using cultivation methods. Magnuson and Lasure ${ }^{28}$ suggested that a rather lower proportion (70-90\%) of soil fungi cannot be obtained using culturing methods. Blackwell ${ }^{29}$ and Taylor et al. ${ }^{30}$ estimated that, including fungi detected by their environmental DNA, the Kingdom Fungi might include between 5.1 and 6 million species worldwide, respectively.

The majority of mycological studies carried out to date on Deception Island have focused on cultivable fungal diversity. Gonçalves et al. ${ }^{31}$ reported seven fungal taxa present in freshwater in Crater Lake, Held and Blanchette ${ }^{32}$ reported 68 taxa on historic wooden structures in Whalers Bay, Figueredo et al. ${ }^{4}$ identified 17 taxa from soil samples from Fumarole Bay and de Menezes et al. ${ }^{33}$ reported 14 taxa from snow. Baeza et al. ${ }^{34}$ used culture-independent techniques to characterize fungal diversity in soils from various different sites in Antarctica, including some samples obtained from the same locations on Deception Island as studied here. They reported 33 taxa, many identified only to genus level, a much lower total than the 346 distinct taxa detected here. Only 10 
$\mathbf{a}$
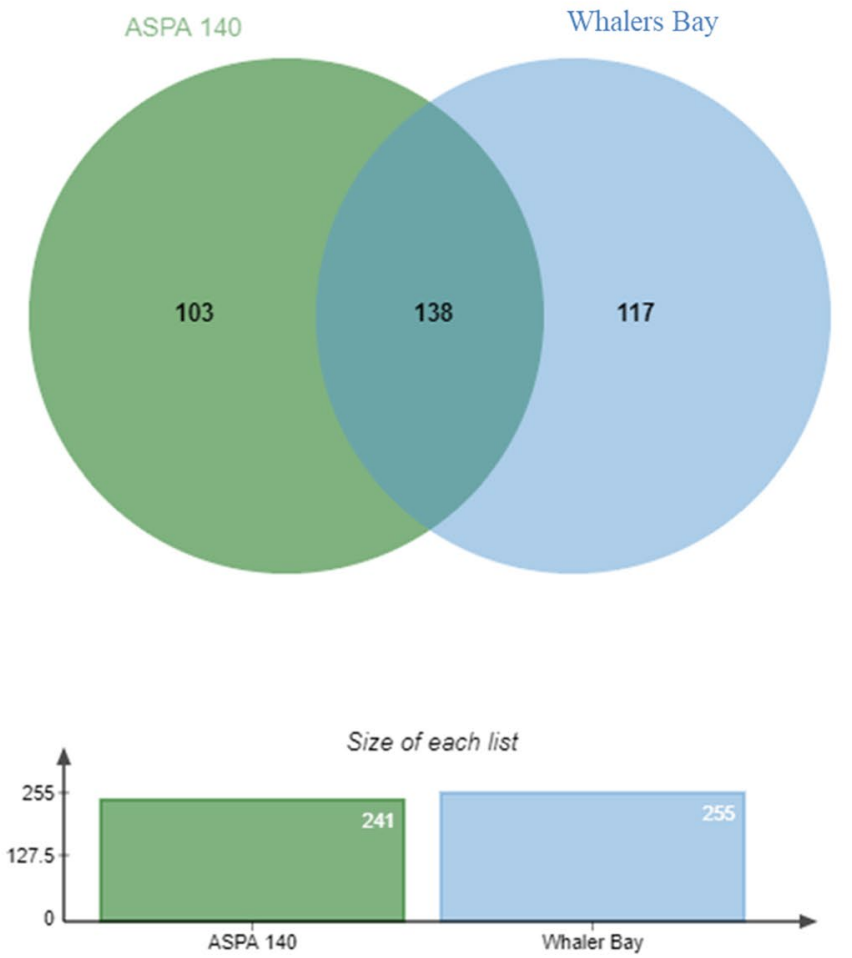

b
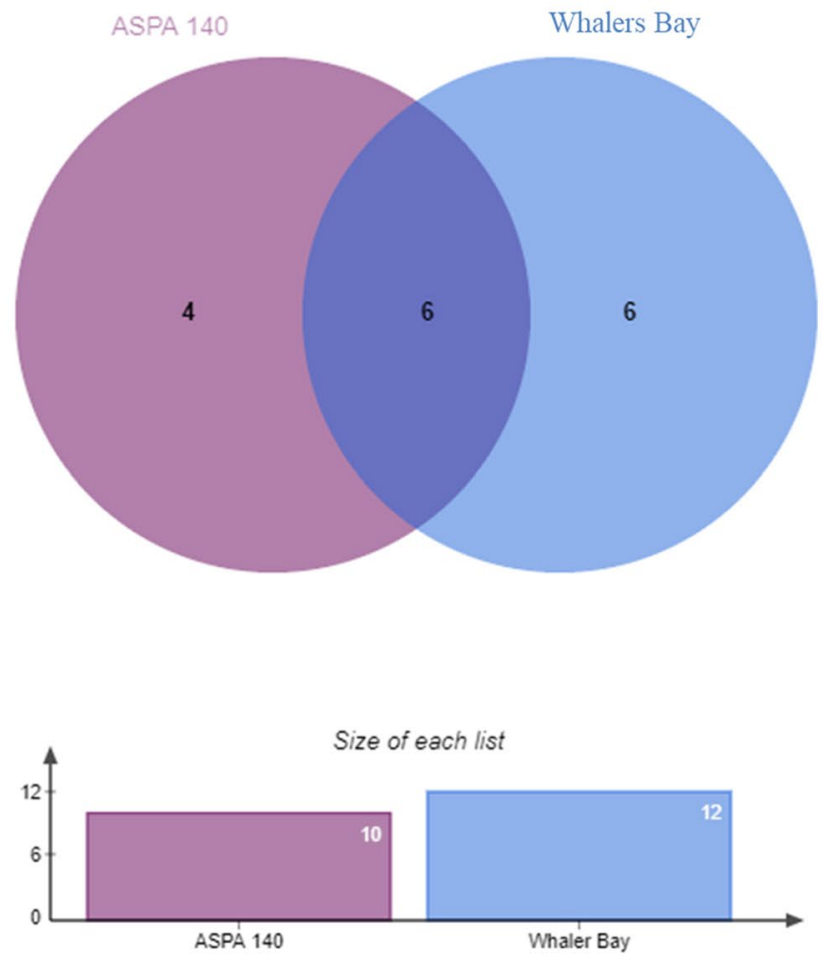

Figure 4. (a) Venn diagram showing the total and (b) dominant (those with $>6000$ reads) fungal taxa distribution between ASPA 140 (non-impacted) and Whalers Bay (impacted) sampling areas.

genera were reported in both studies (Candida, Exophila, Herpotrichia, Lecidea, Malassezia, Merozyma, Pseudogymnoascus, Psoroma, Thelebolus and Verrucaria). Baeza et al. ${ }^{32}$ reported the most abundant taxa to be Verticillium sp., Xanthophyllomyces dendrorhous, Malassezia restricta and Circinaria fruticulosa, differing from the dominant taxa detected in our study (Cladosporium sp., P. roseus, Leotiomycetes sp., Penicillium sp., Mortierella sp. 1, Mortierella sp. 2, P. appendiculatus and Pseudogymnoascus sp.). Our study differs from that of Baeza et al. ${ }^{34}$ in sample size, techniques used, and PCR bias. Despite these differences, our data confirm the presence of a much higher fungal diversity than reported in previous studies. The observation that many of ASVs could only be classified to higher taxonomic levels, with a significant proportion only to the Kingdom Fungi, suggests that it is likely that Antarctica hosts many as yet unrecognised fungal taxa.

Using number of reads as a proxy measure of abundance, Ascomycota was the dominant phylum detected, followed by Basidiomycota, Mortierellomycota and Chytridiomycota. Previous studies of fungal diversity in Antarctic soil have demonstrated the same overall pattern of dominant fungal phyla detected here ${ }^{6,7,24,35,36}$. However, we also detected the presence of taxa from the phyla Mucoromycota and Rozellomycota, which are not commonly reported in Antarctic soils. Although these phyla have global distributions they are poorly known from Antarctica, when compared with Ascomycota, Basidomycota and Mortierellomycota, and are generally regarded as rare ${ }^{2}$.

Members of the genera Cladosporium, Penicillium and Mortierella dominated the assemblages detected in this study. Cladosporium and Penicillium include cosmopolitan species detected in Antarctica. Cladosporium is one of the largest genera of dematiaceous hyphomycetes ${ }^{37}$, with global distribution. It includes species with many different characteristics, including saprophytic and phytopathogenic taxa ${ }^{38}$. In Antarctica, Cladosporium are often associated with the availability of organic matter, such as in moss carpets ${ }^{39,40}$ and the native flowering plant Colobanthus quitensis (Kunth.) Bartl. (Caryophyllaceae) ${ }^{41}$. They are broadly distributed in Antarctica, indicating versatility in adaptation to the extreme conditions of the continent, and have been reported from soil, snow, ice, seawater and marine sediments, freshwater and lake sediments, plants and animals ${ }^{2}$.

Pseudogymnoascus (syn. Geomyces) have been often described from cold habitats of Arctic, alpine, temperate and Antarctic regions ${ }^{2,42-44}$. In Antarctica, Pseudogymnoascus is widely distributed and has been reported from both terrestrial and marine ecosystems, including soils ${ }^{24,42,45}$, mosses ${ }^{39,40,46}$, as an endophyte of C. quitensis ${ }^{41}$, as algicolous fungi of macroalgae ${ }^{47,48}$, in freshwater lakes ${ }^{31}$ and in the lichenosphere ${ }^{49}$. Taxonomic studies of Pseudogymnoascus draw attention to $P$. destructans, causative agent of the lethal disease white-nose syndrome (WNS) in bats of temperate regions ${ }^{50}$. Further studies are required to elucidate if genetic material of this genus detected here belongs to the $P$. destructans group.

The genus Mortierella (Mortierellomycota), whose members are also known as "snow moulds", includes some species often reported in Antarctica. Species of this genus have been reported in association with mosses ${ }^{39,40}$, lichens $^{49}$, soils ${ }^{24,51}$, freshwater ${ }^{31}$, macroalgae ${ }^{52}$ and in the rhizosphere of Deschampsia antarctica Desv. (Poaceae) ${ }^{23}$.

Considering specifically the rare taxa detected in the Deception Island fungal assemblages, the sequence data of several taxa detected from Whalers Bay matched fungi previously reported as opportunistically associated with 


\begin{tabular}{|c|c|c|}
\hline \multirow[b]{2}{*}{ Ecological status } & \multicolumn{2}{|c|}{ Site/number of reads } \\
\hline & ASPA $140^{\mathrm{a}}$ & Whalers Bay \\
\hline \multicolumn{3}{|c|}{ Opportunistic human and animal pathogens } \\
\hline Aphanoascus keratinophilus & 0 & 19 \\
\hline Aspergillus sydowii & 427 & 112 \\
\hline Aspergillus terreus & 160 & 0 \\
\hline Blastobotrys proliferans & 0 & 12 \\
\hline Candida parapsilosis & 0 & 60 \\
\hline Curvularia lunata & 91 & 29 \\
\hline Cutaneotrichosporon smithiae & 0 & 64 \\
\hline Cyphellophora pluriseptata & 24 & 0 \\
\hline Exophiala cancerae & 43 & 0 \\
\hline Magnusiomyces capitatus & 0 & 4 \\
\hline Malassezia dermatis & 36 & 70 \\
\hline Malassezia globosa & 5831 & 689 \\
\hline Malassezia restricta & 11,413 & 3719 \\
\hline Malassezia slooffiae & 35 & 0 \\
\hline Malassezia sympodialis & 364 & 171 \\
\hline Malassezia yamatoensis & 0 & 65 \\
\hline Papiliotrema laurentii & 0 & 358 \\
\hline Pseudallescheria boydii & 21 & 0 \\
\hline Pyrenochaeta keratinophila & 0 & 8 \\
\hline Rhodotorula mucilaginosa & 2565 & 2663 \\
\hline Sporothrix brasiliensis & 0 & 46 \\
\hline Trichosporon asahii & 158 & 68 \\
\hline \multicolumn{3}{|l|}{ Plant pathogens } \\
\hline Aspergillus niger & 292 & 18 \\
\hline Colletotrichum annellatum & 802 & 127 \\
\hline Colletotrichum brevisporum & 0 & 16 \\
\hline Colletotrichum cliviae & 0 & 2524 \\
\hline Curvularia lunata & 91 & 29 \\
\hline Fusarium asiaticum & 0 & 43 \\
\hline Fusarium oxysporum & 0 & 139 \\
\hline Fusarium solani & 0 & 115 \\
\hline Gibberella intricans & 14 & 0 \\
\hline Gibberella tricincta & 4 & 42 \\
\hline Gibberella zeae & 32 & 278 \\
\hline Herpotrichia juniper & 598 & 1074 \\
\hline Mycosphaerella tassiana & 0 & 54 \\
\hline Nigrospora oryzae & 2 & 5 \\
\hline Peniophora albobadia & 5 & 0 \\
\hline Pestalotiopsis trachicarpicola & 0 & 12 \\
\hline Pyrenochaeta keratinophila & 0 & 8 \\
\hline Thanatephorus cucumeris & 23 & 182 \\
\hline Volutella consors & 26 & 0 \\
\hline Cleistothelebolus nipigonensis & 980 & 12,637 \\
\hline \multicolumn{3}{|l|}{ Fungi able to form lichen thalli } \\
\hline Lecidea cancriformis & 129 & 21 \\
\hline Lecidea sp. & 0 & 19 \\
\hline Parmelina sp. & 64 & 0 \\
\hline Placopsis sp. & 35 & 0 \\
\hline Psoroma hypnorum & 24 & 0 \\
\hline Psoroma tenue & 590 & 205 \\
\hline Trimmatothelopsis smaragdula & 73 & 177 \\
\hline Verrucaria alpicola & 2305 & 985 \\
\hline Verrucaria humida & 0 & 24 \\
\hline Verrucaria margacea & 17 & 30 \\
\hline Verrucaria nodosa & 253 & 0 \\
\hline
\end{tabular}

Table 2. Ecological status of the uncultured fungi recovered from different soil samples of Deception Island, Antarctic Peninsula. In bold taxa detected in soil of both sites. ${ }^{\text {a ASPA }}=$ Antarctic Specially Protected Areas. 
humans and animals or able to cause plant diseases. Amongst these, M. dermatis, R. mucilaginosa and T. asahii (human and animal opportunistic) and C. lunata, G. intricans, G. zeae and H. juniper (phytopathogenic) were present in both sampling areas. Although present at apparently low frequency, these fungi merit further attention. For example, de Menezes et al. ${ }^{33}$ reported a high density of cultivable $R$. mucilaginosa in Antarctic snow, a fungus capable of growing at $37^{\circ} \mathrm{C}$ and that displays resistance against the antifungal compound fluconazole, and which may represent a health risk for immunosuppressed persons. In this context, Whalers Bay is a very popular visitor site, including by many elderly tourists likely with weaker immune systems, who may therefore come into contact with the resident microorganisms including those reported as opportunistic disease agents. However, further studies are necessary to assess the risk of infection from resident fungi during a visit to Whalers Bay.

The high-throughput sequencing methodology used in the current study allowed detection of the DNA of a range of fungal taxa able to form the lichenized fungal associations, but without their thalli being visibly present in the soils sampled. Although the lichen diversity of mainland Antarctica and adjacent islands is generally wellknown $^{53}$, that of Deception Island specifically is less well studied, with 70 species currently reported ${ }^{53}$. Among the species whose fungal DNA was detected in the current study, V. alpicola, T. smaragdula, Parmelina sp., V. nodosa, $V$. humida and V. margacea are first records for both Deception Island and Antarctica generally. The dominant DNA detected in both sampled areas was that of V. alpicola. According to Shivarov et al. ${ }^{54}$ this species is known only from Europe (Austria, Great Britain, Germany, Italy, Norway, Romania, Switzerland). Trimmatothelopsis smaragdula is a circumpolar sub-Arctic and alpine species ${ }^{55}$. Verrucaria humida is another European lichen known from Wales, Norway, Germany and Poland, while V. margacea is widespread in Scandinavia, central and western European mountain ranges, and temperate areas in the Southern Hemisphere ${ }^{56}$ and V. nodosa is known only from Wales ${ }^{57}$. Lichens in the genera Psoroma, Lecidea and Placopsis are common in Deception Island and the South Shetland Islands generally.

\section{Conclusions}

DNA metabarcoding of soil fungal assemblages in samples obtained from ASPA 140 subsite B and Whalers Bay on Deception Island indicated the presence of a rich fungal diversity. The 'rare' fungal taxa detected in both areas included fungi reported as human and animal opportunistic and plant pathogens. The diversity detected may have been transported to Deception Island associated with human activities such as the historic whaling industry, research, tourism, through natural transport by birds or in the air column, or represent resident fungi not previously described. Further long-term studies are required to elucidate how biological colonization of the island may be affected by climatic changes and other anthropogenic influences.

Received: 18 June 2020; Accepted: 29 October 2020

Published online: 15 December 2020

\section{References}

1. Convey, P. et al. The spatial structure of Antarctic biodiversity. Ecol. Monogr. 84, 203-244 (2014).

2. Rosa, L. H. et al. Fungi in Antarctica: diversity, ecology, effects of climate change, and bioprospection for bioactive compounds. In Fungi of Antarctica: Diversity, Ecology and Biotechnological Applications (ed. Rosa, L. H.) 1-18 (Springer, Berlin, 2019).

3. Hart, I. B. Whaling in the Falkland Islands Dependencies, 1904-1931 (Newton St. Margarets, Pequena, 2006).

4. Figueredo, H. M. et al. Diversity and ecology of cultivable fungi isolated from the thermal soil gradients in Deception Island Antarctica. Extremophiles 24, 219-225 (2020).

5. Smellie, J. L. Lithostratigraphy and volcanic evolution of Deception Island South Shetland Islands. Antarct. Sci. 13, 188-209 (2001).

6. Fell, J. W. et al. Biodiversity of micro-eukaryotes in Antarctic dry valley soils with <5\% soil moisture. Soil Biol. Biochem. 38, 3107-3119 (2006).

7. Godinho, V. M. et al. Diversity and bioprospection of fungal community present in oligotrophic soil of continental Antarctica. Extremophiles 19, 585-596 (2015).

8. Chen, S. et al. Validation of the ITS2 region as a novel DNA barcode for identifying medicinal plant species. PLoS ONE 5, e8613 (2010).

9. Richardson, R. T. et al. Application of ITS2 metabarcoding to determine the provenance of pollen collected by honey bees in an agroecosystem. Appl. Plant Sci. 3, 1400066 (2015).

10. White, T. J. et al. Amplification and direct sequencing of fungal ribosomal RNA genes for phylogenetics. In PCR Protocols: A Guide to Methods and Applications (eds Innis, M. A. et al.) 315-322 (Academic Press, New York, 1990).

11. Joshi, N. A., Fass, J. N. Sickle: A Sliding-Window, Adaptive, Quality-Based Trimming Tool for FastQ files (Version 1.33) [Software]. https://github.com/najoshi/sickle. Accessed June 2020 (2011).

12. Bolyen, E. et al. Reproducible, interactive, scalable and extensible microbiome data science using QIIME 2. Nat. Biotechnol 37, 852-857 (2019).

13. Callahan, B. J. et al. Holmes SP. DADA2: high-resolution sample inference from Illumina amplicon data. Nat. Methods 13, 581-583 (2016).

14. Bokulich, N. A. et al. Optimizing taxonomic classification of marker-gene amplicon sequences with QIIME 2's q2-feature-classifier plugin. Microbiome 6, 90 (2018).

15. Kõljalg, U. et al. Towards a unified paradigm for sequence-based identification of fungi. Mol. Ecol. 22, 5271-5277 (2013).

16. Medinger, R. et al. Diversity in a hidden world: potential and limitation of next-generation sequencing for surveys of molecular diversity of eukaryotic microorganisms. Mol. Ecol. 19, 32-40 (2010).

17. Weber, A. A. \& Pawlowski, J. Can abundance of protists be inferred from sequence data: a case study of Foraminifera. PLoS ONE 8, e56739 (2013).

18. Giner, C. R. et al. Environmental sequencing provides reasonable estimates of the relative abundance of specific picoeukaryotes. Appl. Environ. Microbiol. 82, 4757-4766 (2016).

19. Deiner, K. et al. Environmental DNA metabarcoding: transforming how we survey animal and plant communities. Mol. Ecol. 26, 5872-5895 (2017).

20. Hering, D. et al. Implementation options for DNA-based identification into ecological status assessment under the European Water Framework Directive. Water Res. 138, 192-205 (2018). 
21. Hammer, Ø., Harper, D. A. T. \& Ryan, P. D. PAST: paleontological statistics software package for education and data analysis. Palaeontol. Electron. 4, 9 (2001).

22. Bardou, P. et al. An interactive Venn diagram viewer. BMC Bioinform. 15, 293 (2014).

23. Gonçalves, V. N. et al. Antibacterial, antifungal and antiprotozoal activities of fungal communities present in different substrates from Antarctica. Polar Biol. 38, 1143-1152 (2015).

24. Gomes, E. C. et al. Cultivable fungi present in Antarctic soils: taxonomy, phylogeny, diversity, and bioprospecting of antiparasitic and herbicidal metabolites. Extremophiles 22, 381-393 (2018).

25. Bridge, P. D. \& Spooner, B. M. Non-lichenized Antarctic fungi: transient visitors or members of a cryptic ecosystem?. Fungal Ecol. 5, 381-394 (2012).

26. Amann, R. I., Ludwig, W. \& Schleifer, K. H. Phylogenetic identification and in situ detection of individual microbial cells without cultivation. Microbiol. Rev. 59, 143-169 (1995).

27. Rappe, M. S. \& Giovannoni, S. J. The uncultured microbial majority. Annu. Rev. Microbial. 57, 369-394 (2003).

28. Magnuson, J. K., Lasure, L. L. Fungal diversity in soils as assessed by direct culture and molecular techniques. In Salt Lake: Abstracts from the 102nd General Meeting of the American Society for Microbiology, 19-23. http://www.pnnl.gov/biobased/docs/funga 1_diversity.pdf. Accessed June 2020 (2002).

29. Blackwell, M. The fungi: 1,2,3...5,1 million species?. Am. J. Bot. 98, 426-438 (2011).

30. Taylor, D. E. et al. A first comprehensive census of fungi in soil reveals both hyperdiversity and fine-scale niche partitioning. Ecol. Monogr. 84, 3-20 (2014).

31. Gonçalves, V. N. et al. Diversity and distribution of fungal communities in lakes of Antarctica. FEMS Microbiol. Ecol. 82, 459-471 (2012).

32. Held, B. W. \& Blanchette, R. A. Deception Island, Antarctica, harbors a diverse assemblage of wood decay fungi. Fungal Biol. 121, $145-157$ (2017).

33. de Menezes, G. C. A. et al. Diversity, distribution, and ecology of fungi in the seasonal snow of Antarctica. Microorganisms 7, 445 (2019).

34. Baeza, M. et al. Amplicon-metagenomic analysis of fungi from Antarctic terrestrial habitats. Front. Microbiol. 8, 2235 (2017).

35. Boyd, W. L., Staley, J. T. \& Boyd, J. W. Ecology of soil microorganisms of Antarctica. Antarctic soils and soil forming processes. Antarct. Res. 8, 125-129 (1966).

36. Adams, B. J. et al. Diversity and distribution of Victoria Land biota. Soil Biol. Biochem. 38, 3003-3018 (2006).

37. Bensch, K. et al. Species and ecological diversity within the Cladosporium cladosporioides complex (Davidiellaceae, Capnodiales). Stud. Mycol. 67, 1-94 (2010).

38. Torres, D. E. et al. Cladosporium cladosporioides and Cladosporium pseudocladosporioides as potential new fungal antagonists of Puccinia horiana Henn., the causal agent of chrysanthemum white rust. PLoS ONE 12, e0170782 (2017).

39. Tosi, S., Casado, B. \& Gerdol, R. Fungi isolated from Antarctic mosses. Polar Biol. 25, 262-268 (2002).

40. Rosa, L. H. et al. Opportunistic fungal assemblages present on fairy rings spread on different moss species in the Antarctic Peninsula. Polar Biol. 43, 587-596 (2020).

41. Rosa, L. H. et al. Endophytic fungi community associated with the dicotyledonous plant Colobanthus quitensis (Kunth) Bartl. (Caryophyllaceae) in Antarctica. FEMS Microbiol. Ecol. 73, 178-189 (2010).

42. Mercantini, R., Marsella, R. \& Cervellati, M. C. Keratinophilic fungi isolated from Antarctic soil. Mycopathologia 106, 47-52 (1989).

43. Lorch, J. M. et al. A culture-based survey of fungi in soil from bat hibernacula in the eastern United States and its implications for detection of Geomyces destructans, the causal agent of bat white-nose syndrome. Mycologia 105, 237-252 (2013).

44. Minnis, A. M. \& Lindner, D. L. Phylogenetic evaluation of Geomyces and allies reveals no close relatives of Pseudogymnoascus destructans, comb. nov., in bat hibernacula of eastern North America. Fungal Biol. 117, 638-649 (2013).

45. Arenz, B. E. \& Blanchette, R. A. Distribution and abundance of soil fungi in Antarctica at sites on the Peninsula, Ross Sea Region and McMurdo Dry Valleys. Soil Biol. Biochem. 43, 308-315 (2011).

46. Carvalho, C. R. et al. Fungi associated with the briosphere of the bipolar mosses Polytrichastrum alpinum and Polytrichum juniperinum in Antarctica. Polar Biol. 43, 545-553 (2020).

47. Loque, C. P. et al. Fungal community associated with marine macroalgae from Antarctica. Polar Biol. 33, 641-648 (2010).

48. Furbino, L. E. et al. Diversity patterns, ecology and biological activities of fungal communities associated with the endemic macroalgae across the Antarctic. Microb. Ecol. 67, 775-787 (2014)

49. Santiago, I. F. et al. Lichenosphere: a protected natural microhabitat of the non-lichenised fungal communities living in extreme environments of Antarctica. Extremophiles 19, 1087-1097 (2015).

50. Lorch, J. M. et al. Experimental infection of bats with Geomyces destructans causes white-nose syndrome. Nature 480, 376-378 (2011).

51. Bridge, P. D. \& Newsham, K. K. Soil fungal community composition at Mars Oasis, a southern maritime Antarctic site, assessed by PCR amplification and cloning. Fungal Ecol. 2, 66-74 (2009).

52. Godinho, V. M. et al. Diversity and bioprospecting of fungal communities associated with endemic and cold-adapted macroalgae in Antarctica. ISME J. 7, 1434-1451 (2013).

53. Øvestedal, D. O. \& Smith, R. I. L. Lichens of Antarctica and South Georgia: A Guide to Their Identification and Ecology (Cambridge University Press, Cambridge, 2001)

54. Shivarov, V. V., Thüs, H. \& Denchev, C. M. First records of two freshwater lichens, Hydropunctaria scabra and Verrucaria alpicola, from Bulgaria. Mycobiota 7, 1-5 (2017).

55. Thompson, J. American Arctic Lichens: The Microlichens. https://lichenportal.org/cnalh/taxa/index.php?taxon $=12767$ 0\&clid=1019. Accessed June 2020 (1997).

56. Thüs, H. et al. Revision of the Verrucaria elaeomelaena species complex and morphologically similar freshwater lichens (Verrucariaceae, Ascomycota). Phytotaxa 197, 161-185 (2015).

57. Orange, A. Four new species of Verrucaria (Verrucariaceae, lichenized Ascomycota) from freshwater habitats in Europe. Lichenologist 45, 305-322 (2013).

\section{Acknowledgements}

This study received financial support from CNPq, PROANTAR, FAPEMIG, Coordenação de Aperfeiçoamento de Pessoal de Nível Superior-Brasil (CAPES), INCT Criosfera 2. P. Convey is supported by NERC core funding to the British Antarctic Survey's 'Biodiversity, Evolution and Adaptation' Team. We are also grateful for the generous support of the Spanish Polar Committee and its staff at Gabriel de Castilla base.

\section{Author contributions}

L.H.R. and P.E.A.S.C. conceived the study. T.H.S. and M.B.O. performed DNA extraction from soils. L.H.R., P.E.A.S.C., O.H.B.Z., M.S., P.C., M.C.S., C.A.R. analyzed the results and wrote the manuscript. All authors read and approved the final manuscript. 


\section{Competing interests}

The authors declare no competing interests.

\section{Additional information}

Supplementary Information The online version contains supplementary material available at https:/doi. org/10.1038/s41598-020-78934-7.

Correspondence and requests for materials should be addressed to L.H.R.

Reprints and permissions information is available at www.nature.com/reprints.

Publisher's note Springer Nature remains neutral with regard to jurisdictional claims in published maps and institutional affiliations.

(c) (1) Open Access This article is licensed under a Creative Commons Attribution 4.0 International License, which permits use, sharing, adaptation, distribution and reproduction in any medium or format, as long as you give appropriate credit to the original author(s) and the source, provide a link to the Creative Commons licence, and indicate if changes were made. The images or other third party material in this article are included in the article's Creative Commons licence, unless indicated otherwise in a credit line to the material. If material is not included in the article's Creative Commons licence and your intended use is not permitted by statutory regulation or exceeds the permitted use, you will need to obtain permission directly from the copyright holder. To view a copy of this licence, visit http://creativecommons.org/licenses/by/4.0/.

(C) The Author(s) 2020 